\title{
Retinyl acetate mediates autocrine proliferation and wound healing of keratinocytes through a c-neu (erbB-2)-like receptor
}

\begin{abstract}
Studies were initiated on retinyl acetate, a natural fatty acid retinol ester, to determine its effect on the clonal growth of normal human keratinocytes (NHK) and on the proliferation of $\mathrm{HaCaT}$ immortalized human keratinocytes. Both can be propagated in a chemically-defined serum-free medium. Retinyl acetate (RetAc) at physiological concentrations supports the autocrine production of an EGF-like growth factor that stimulates proliferation of both normal and $\mathrm{HaCaT}$ cells in serum-free media supplemented only with insulin. The possible cellular mechanism of autocrine stimulated proliferation induced by RetAc was elucidated by inhibiting clonal growth following treatment with a specific receptor tyrosine phosphokinase inhibitor, and by employing indirect immunofluorescence antibody detection microscopy to positively stain $c$-neu (erbB-2) cell membrane targets. C-neu antibody positively stains the cytoplasm of untreated keratinocytes, which is relocalized to focal adhesion cell surface receptors after alkaline phosphatase treatment, indicating a phosphorylationlabile receptor. By contrast, RetAc-treated keratinocytes stained positively for c-neu at cell surface focal adhesion sites that were labile to dephosphorylation by treatment with alkaline phosphatase. In addition, we investigated the effect of retinyl acetate on wound healing in an epidermal monolayer wound healing model. RetAc inhibited wound closure of damaged epidermal sheets in the wound healing zone at concentrations greater than those that stimulate keratinocyte proliferation. This result and other previous studies indicate RetAc stimulation of keratinocyte proliferation can be employed without EGF to produce a cultured stratified squamous keratinized epidermis suitable for wound healing applications.
\end{abstract}

Keywords: clonal growth, $c$-neu proto-oncogene, EGF, HaCaT cells, keratinocytes, retinyl acetate, wound healing
Volume 4 Issue 2 - 2018

\author{
John JWille,' Jong Y Park, ${ }^{2}$ \\ 'Bioplast Medical LLC, Department of Cell Biology, USA \\ ${ }^{2}$ Department of Cancer Epidemiology, Moffitt Cancer Center, \\ USA
}

Correspondence: John JWille, Bioplast Medical LLC, Department of Cell Biology, 9 Chesterfield Georgetown Rd., Chesterfield, New Jersey 085I5, USA, Tel +6092 6114 88, Fax 60929119 97, Email jjwille@aol.com

Received: January 29, 2018 | Published: April 10, 2018

\section{Introduction}

Pleiotropic effects of retinoids on cell growth signal transduction and receptor-mediated gene regulation are well documented. ${ }^{1,2}$ The use of chemically-defined serum free medium (SFM) has identified two protein growth factors, EGF and insulin, that are required for normal human keratinocytes $(\mathrm{NHK})$ proliferation in low calcium $(<1 \mathrm{mM})$ SFM medium. ${ }^{3,4}$ The role of retinoids in regulating proliferation of normal human keratinocytes is less well-understood. For example, all-trans retinoic acid (t-RA) stimulates proliferation in essential fatty acid-supplemented keratinocytes. ${ }^{5}$ Retinoic acid (t-RA, $10^{-7} \mathrm{M}$ ) treatment inhibited proliferation of $\mathrm{HaCaT}$ cells adapted to serumfree DME/Hams F12 medium, while retinol (ROL, $10^{-7} \mathrm{M}$ ) did not, but overall growth in this SFM was much curtailed. ${ }^{6}$ By contrast, a panel of retinoids including t-RA, ROL, 13-cis RA, all inhibited the clonal growth of NHK in SFM supplemented with EGF and insulin in rapidly proliferating NHK. ${ }^{7}$ The strength of inhibition was linearly correlated with their ability to suppress both ornithine decarboxylase enzyme induction by tumor promoter and papilloma formation in the mouse skin model of tumorigenesis. ${ }^{7}$ By contrast, t-RA stimulates growth-arrested adult keratinocytes in protein growth factor-deficient SFM. ${ }^{8}$ These conflicting effects appear to involve differing growth media conditions. Verani et al. ${ }^{9}$ also reported that induced alterations in membrane intracellular calcium ion fluctuations appear to underlie retinoid growth stimulation. ${ }^{9}$ Retinoid stimulation of growth-arrested
NHK was also found to involve autocrine production of a heparinbonding EGF (hb-EGF), and activation of erbB receptors ${ }^{10}$ present on suprabasal cells. Moreover, autocrine stimulation via these signaling pathways appears to underlie retinoid-induced epidermal hyperplasia. ${ }^{10}$ Recently, t-RA was reported to inhibit the expression of the erbB (c-neu) receptor and other proto-oncogenes in several different epidermoid carcinoma cell lines..$^{11}$ In addition, t-RA reverses the super-induction by alcohol of aryl hydrocarbon hydrylase induced by benzo $(\alpha)$ pyrene in SFM culture of NHK. ${ }^{12}$ Retinoids also have profound effects on epidermal keratinocyte differentiation in many different tumor cells lines. ${ }^{13}$ Retinyl acetate (RetAc) is a naturally occurring fatty acid ester of retinol, and is less toxic than t-RA. It is stored in human liver and is involved to metabolic conversion to vitamin A (retinol). Here, we explore the effect of RetAc on the clonal growth of $\mathrm{NHK}$ and $\mathrm{HaCaT}$ cells treated with different combinations of EGF and insulin. We established that serum can be dispensed with by culturing $\mathrm{HaCaT}$ keratinocytes in SFM supplemented with EGF and insulin. This allowed us to conduct detailed clonal assay to determine their minimal growth factor requirements. Clonal growth assay studies also examined the effect of RetAc. Unlike t-RA, ${ }^{7}$ we report that RetAc stimulates $\mathrm{HaCaT}$ clonal growth at physiological levels. We further explored the underlying biochemical events that reveal an autocrine signaling pathway and possible involvement of the $c-n e u$ (erbB-2) cytoplasmic receptor and a treatment-induced translocation to focal membrane areas. Finally, we examined the effect 
of RetAc on wound healing using in an in vitro epidermal keratinocyte wound healing model. ${ }^{14}$

\section{Materials and methods}

\section{Materials}

All chemicals were obtained from Sigma-Aldrich Company (St Louis, MO). erbB (c-neu) antibodies and anti-EGF antibodies were purchased from Oncogene Sciences (Manhasset, NY).

\section{Cell cultures}

Normal human neonatal foreskin keratinocytes were prepared and maintained in serum free MCDB 153 medium as previously described. ${ }^{3}$ $\mathrm{HaCaT}$ cells, a spontaneously immortalized cell line were donated by Dr N. Fusenig, German Cancer Institute (Berlin, Germany). They were routinely maintained by serial passage in DMEM: $10 \%$ fetal calf serum. In preliminary studies, we determined that $\mathrm{HaCaT}$ cells can be adapted to growth in serum-free MCDB 153 medium supplemented with EGF $(5 \mathrm{ng} / \mathrm{ml})$ and insulin $(5 \mu \mathrm{g} / \mathrm{ml})$. For clonal growth assays, $\mathrm{HaCaT}$ cells were washed three times with $3 \mathrm{~mL}$ aliquots of ice-cold basal nutrient MCDB 153 medium, seeded in to sterile $35 \mathrm{~mm}^{2}$ plastic disposable Petri dishes, and refed various combinations of growth factors and RetAc. The dishes were incubated at $37^{\circ} \mathrm{C}$ for 10 days and the cells fixed with $50 \%$ alcohol and stained with $0.2 \%$ crystal violet and photographed previously described. ${ }^{4}$

\section{Immunofluorescence -antibody staining}

The technique of indirect immunofluorescence (IIF) was performed according to previous studies. ${ }^{14}$ Briefly, we performed cytochemistry, using the $c-n e u$ antibody as follows: normal human neonatal foreskin keratinocytes were first cultured on circular glass chips. At the desired time, the chips were placed in to clean glass Petri plates and the cells fixed with drops of 3:1 acetone: water fixative and air dried. Antibody solutions were applied at the manufacturer's recommended titers for cytochemistry applications and applied to the fixed cells for 30 minutes at room temperature. To prevent adventitious binding of $c-n e u$ antibody, the fixed cells were prewashed with a $0.1 \%$ ice-cold solution of BSA, followed by several saline washes. To detect c-neu binding a second rat anti-mouse $\operatorname{IgG}$ polyclonal antibody conjugated with fluorescin directed against the $c$-neu mouse monoclonal antibody was applied and incubated for an additional 30 minutes. The binary antibody- cell complex was rinsed several times with saline and permanent glass slides were prepared for microscopic viewing and analysis using a UV-epifluorescence Nikon phase compound microscope equipped with a Cannon microphotographic camera system.

\section{Wound healing studies}

$\mathrm{HaCaT}$ cells were plated in sterile $35 \mathrm{~mm}^{2}$ plastic disposable petro dishes at $5 \times 10^{3} \mathrm{cells} / \mathrm{cm}^{2}$ and refed SFM containing EGF $(5 \mathrm{ng} / \mathrm{ml})$ and insulin $(5 \mathrm{mg} / \mathrm{ml})$ every other day until the cultures reached confluence. The cells were washed $3 \mathrm{X}$ with ice-cold basal nutrient MCDB 153 medium and the dishes refed fresh SFM supplemented with insulin and varying concentrations of RetAc. Control dishes were refed SFM supplemented with EFG and insulin. Immediately thereafter, the control and RetAc-treated confluent epidermal keratinocyte sheets were wounded by making a $2.5 \mathrm{~mm}$ wide scratch along the midline diameter with a fine closed tip $9 \mathrm{~cm}$ sterile glass Pasteur pipette as previously described. ${ }^{15}$ The dishes were then placed in $\mathrm{CO}_{2}$ gas humidified incubator at $37^{\circ} \mathrm{C}$ for 24 hours, fixed, stained with $0.2 \%$ crystal violet stain and photographed. Percent closure of the healed wound area was measured with a 10X eyepiece micrometer ruler and $4 X$ phase objective with a Nikon Diaphot phase contrast microscope.

\section{Results}

Preliminary studies established that serially- passaged cultures of $\mathrm{HaCaT}$, an immortalized cell line, previously maintained in DMEM: 10\% FCS can be cultured in serum-free MCDB 153 medium supplemented with insulin $(5 \mathrm{mg} / \mathrm{ml})$ and EGF $(5 \mathrm{ng} / \mathrm{ml})$. The question arose whether it could also be cultured in a protein growth factordeficient SFM supplemented with RetAc medium.

Figure 1 is an image showing the morphological appearance of $\mathrm{HaCaT}$ cells cultured in SFM supplemented with EGF and insulin (A) compared with SFM cultures supplemented with RetAc and insulin only (B). RetAc-treated cultures appear compact resembling HaCaT cultures grown in serum-containing media containing millimole concentrations of calcium ions.

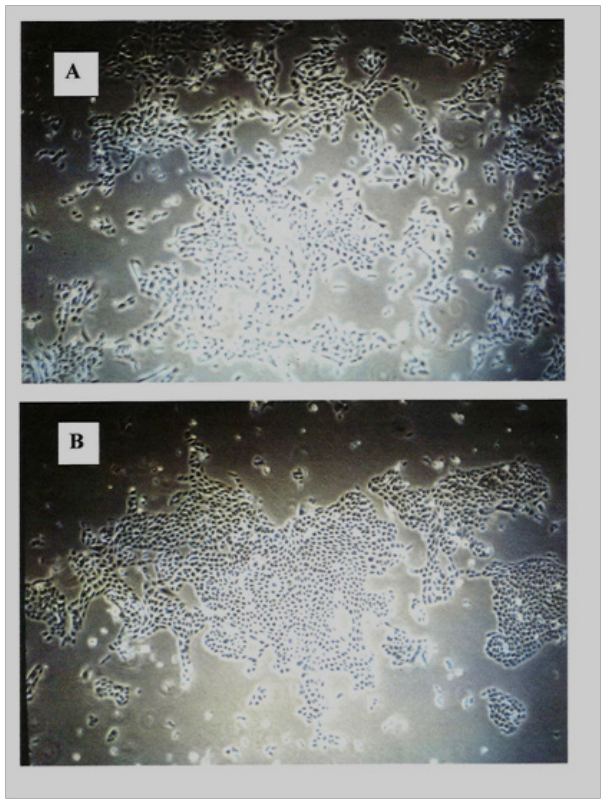

Figure I Phase contrast image showing the typical culture morphology of subconfluent cultures of $\mathrm{HaCat}$ keratinocytes cultured in (A) growth factor -replete SFM or in (B) growth-factor-deficient SFM supplemented with RetAc and insulin. RetAc-treated cultures appear more compact than EGF and insulin -treated cultures. Scale bars shown in white are 50 microns.

Figure 2 shows the clonal growth of $\mathrm{HaCaT}$ cells grown in SFM supplemented with insulin and EGF, fixed and stained one day after seeding at $5 \times 10^{3}$ cells $/ \mathrm{cm}^{2}$ (A) compared with clonal growth achieved in SFM supplemented with insulin and EGF and fixed and stained 10 days later (B) compared to enhanced clonal growth achieved after 10 days when HaCaT was grown in SFM supplemented with insulin and RetAc (C). The result show that RetAc-treated keratinocytes display enhanced clonal growth relative to EGF plus insulin supplemented cultures

Further clonal growth studies, explored growth factor control of $\mathrm{HaCaT}$ cells. Induction of autocrine proliferation is validated by the fact that no growth occurs in growth factor-deficient SFM or in SFM supplemented with insulin $(5 \mu \mathrm{g} / \mathrm{ml})$ only, while enhanced 
clonal growth is observed in standard medium supplemented with $5 \mu \mathrm{g} / \mathrm{ml}$ insulin plus $3 \times 10^{-8} \mathrm{M}$ RetAc. The moderate clonal growth often observed in SFM supplemented with insulin it might be due to carry-over of EGF or incomplete removal of EGF from cell surface adherence. The above results suggest that RetAc stimulates autocrine production of an EGF-like activity that subsequently binds to EGF receptors. This hypothesis was investigated using a selective inhibitor of receptor tyrosine protein phosphokinase (RTK1, PD98059) on the enhanced clonal growth of $\mathrm{HaCaT}$ cell stimulated to proliferate by RetAc.

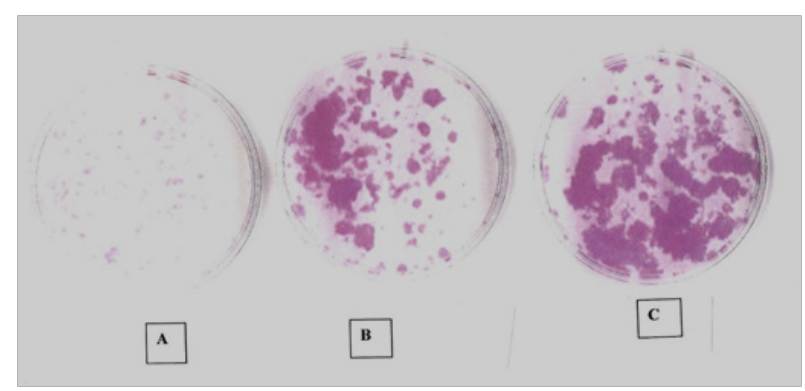

Figure 2 Photographic image of keratinocyte cultured under differing clonal growth conditions. Keratinocytes were seeded at $5 \times 103 \mathrm{cell} / \mathrm{sm} 2$ on day refed protein growth factors(EGF and insulin) SFM and fixed and stained one day later (A) and 10 days later (B) compared to (C), keratinocytes refed SFM supplmeneted with RetAc and insulin on day one and fixed and stained 10 days later. Magnification factor, $1 / 2 X$.

Figure 3 shows that clonal growth in SFM supplemented with insulin in the presence of $2 \mu \mathrm{M}$ of RTK1 (C) was inhibited relative to clonal growth achieved during the same day of growth in serum-free medium containing both insulin and RetAc without RTK1 (B), and was less than clonal growth as $\mathrm{HaCaT}$ cells refed SFM supplemented with RetAc and insulin and fixed and stained one day earlier (A).

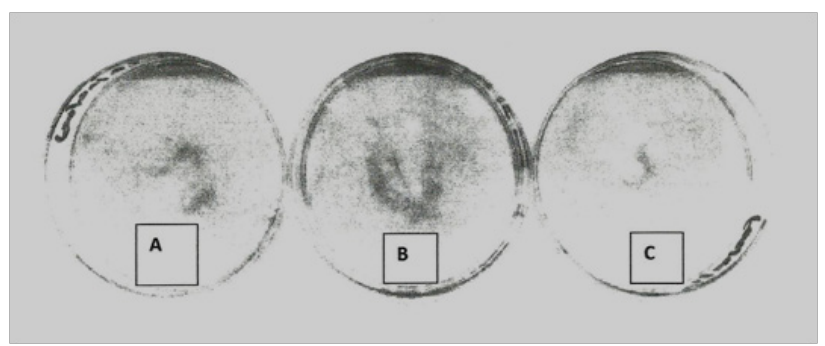

Figure 3 Photographic image of keratinocyte cultured under differing clonal growth conditions. Keratinocytes were seeded at $5 \times 103$ cells $/ \mathrm{cm} 2$ on day one and refed protein growth factors-deficient SFM supplemented with RetAc and insulin. (A) cells were fixed and stained four day later or (B) 5 days later. (C), keratinocytes were refed SFM supplmeneted with RetAc and insulin on day one and on day4 refed SFM supplemented with RetAc plus RTKI and fixed and stained one day later. Dark material inside each dishe are stained keratinocytes, Magnification of dishes, $\mathrm{I} / 2 \mathrm{X}$.

These results suggest that retinoid-induced clonal growth of $\mathrm{HaCaT}$ keratinocytes is dependent on phosphorylation of the tyrosine residue in an activated EGF-like growth factor receptor protein required for downstream signal transduction and phosphorylation events necessary for cell proliferation. To examine this possibility, we investigated the effect of RetAc-treatment on erbB-2(c-neu) expression using the technique of IIF microscopy detection of c-neu antibodies. Preliminary IIF studies, showed that c-neu antibody reacted positively only with keratinocytes cells in early G1 phase of the cell cycle or with post-mitotic suprabasal keratinocytes (data not shown). Figure 4 (A) shows that $c$-neu staining was dispersed over the entire cytoplasm with a tendency toward monopolar distribution of finely and particulate stained foci. Figure 4 (B) shows that NHK cells treated with alkaline phosphatase enzyme (AP) prior to staining in order to remove phosphate groups from proteins before straining with c-neu antibody stain monopolar membrane located infocal adherence areas with loss of generalized cytoplasmic staining. Focal adherence plaques are areas where presumptive cell-to-cell contacts occur.

We infer that in keratinocyte cells phosphorylation of c-neu receptors are distributed away from areas of cell-to-cell contact, while de-phosphorylation by AP reveals that unphosphorylated c-neu-like receptors are primarily located at specialized membrane monofocal plaques, a situation that prevails in proliferating keratinocytes cultures. The effect of RetAc on c-neu expression in keratinocyte cultures propagated in SFM was also examined by IIF microscopy and detection of c-neu antibody staining as seen in Figure 4 (C). For the RetAc-treated cells the $c-n e u$ stable phosphorylation state is restricted to monopolar focal adherence areas. Figure 4 (D) shows that these membrane located $c$-neu phosphorylation sites are lost after AP treatment.
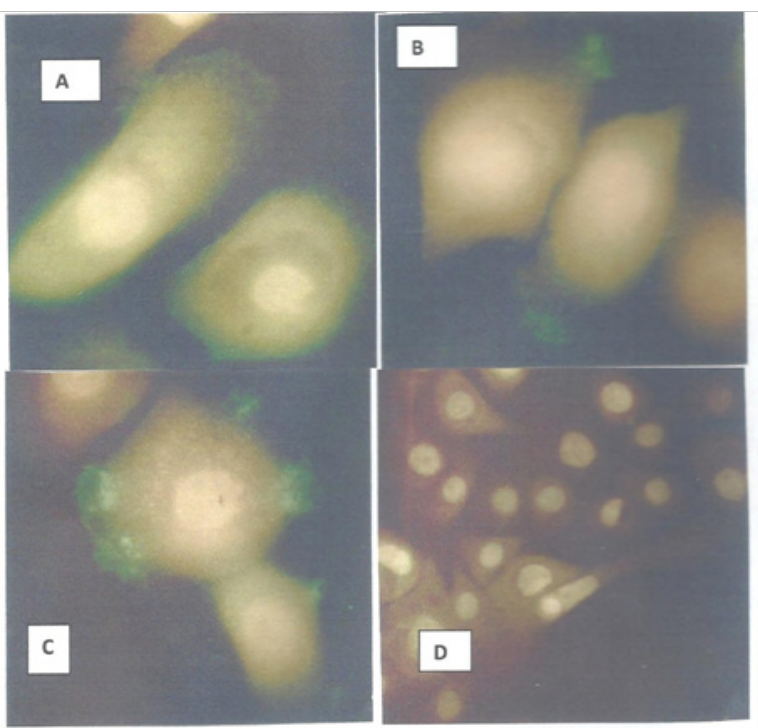

Figure 4 Photomicrograph images showing result of c-neu antibody and IIF microscopy of keratinocytes cultured under different conditions. Note positive fluorescence is shown in green color; nuclei were counterstained yellow-red with propidium iodide (A) growth factor replete SFM prepared in the absence of alkaline phosphatase enzyme pretreatment or (B) with alkaline phosphatase enzyme pretreatment; keratinocyte were cultured in growth factor-deficient SFM supplemented with RetAc and insulin and stained with c-neu antibody in: (C) absence of alkaline phosphatase enzyme pretreatment or (D) with alkaline phosphatase enzyme treatment. Scale bars in white for A, $B$ and $C$ are $I$ micron, and for $D$ is 3.5 microns.

We next examined the effect of RetAc on HaCaT keratinocytes on healing of in vitro wounded epithelial keratinocyte sheets under autocrine growth conditions. Confluent cultures were washed with ice-cold basal MCDB 153 SFM and refed a SFM, and subjected to wound healing as described (Methods). Figure 5(D) is a photographic image of a visible linear wound scar designated here as a wound healing zone (WHZ). Figure 5(A) shows that wounds of this diameter routinely repaired the breach in the WHZ in 24 hours in growth factor replete SFM. By contrast, wound healing was delayed when confluent 
cultures were cultured in growth factor deficient SFM supplemented with RetAc $\left(3 \times 10^{-7} \mathrm{M}\right)$ and insulin $(\mathrm{B})$, considerably less when keratinocyte monlayers were cultured in SFM with insulin and $3 \times 10$ ${ }^{6} \mathrm{M}(\mathrm{C})$ no healing when cultured at toxic levels of RetAc $\left(3 \times 10^{-5} \mathrm{M}\right)$ (D).

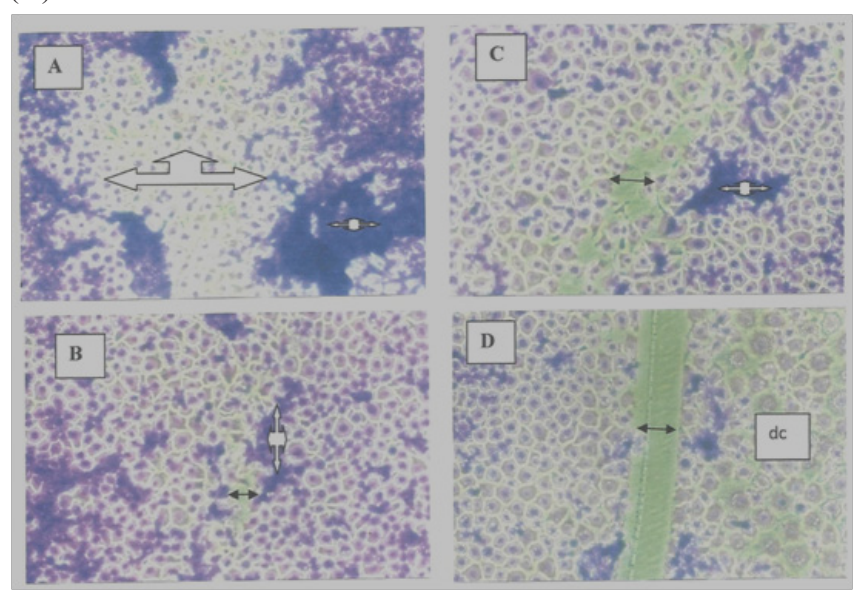

Figure 5 (A) complete healing in the wound healing zone shown by the triple headed arrow, (B) nearly complete healing in the presence of $3 \times 10-7 M$ RetAc, (C) partial to complete healing in the presence of $3 \times 10-6 \mathrm{M}$, and (D) no healing in the presence of $3 \times 10-5 \mathrm{M}$ retinyl acetate. Double -headed black arrows in $B, C$ and $D$ show extent of closure in WHZ. White quad-arrow in $B$ and $C$ show remnants of dead cells removed from the WHZ. Box designated dc (dead cells) show field of vacuolated dead cells. Scale bars in white are 2.5 $\mathrm{mm}$ wide.

\section{Discussion}

We presented data demonstrating that RetAc at physiological concentrations support sub-confluent monolayer keratinocyte proliferation in a growth factor-deficient SFM supplemented with insulin only. The use of RetAc avoids the costly use of EGF as a necessary protein growth factor requirement. These results are consistent with ones from a previous study, ${ }^{8}$ and with earlier reports ${ }^{16,17}$ identifying a heparin binding EGF-like growth factor as the target in the intercellular regulation of epidermal basal cell growth by suprabasal retinoic acid receptors. Confluent cultures of NHK are known to secrete amphiregulin, an autocrine produced EGFlike heparin-binding EGF-like family of growth factors..$^{18}$ Moreover, confluent monolayers of growth-arrested adult keratinocyte can be propagated in millimole concentrations of calcium $(1.4 \mathrm{mM})$ in SFM deficient in EGF treated concomitantly with t-RA (0.1-2.5ng/ $\mathrm{ml}){ }^{8}$ The effect was relatively small, approximately $35 \%$ of the maximal proliferation rate observed in growth factor supplemented medium. Also, the optimal concentration of t-RA required to produce the effect was $0.5 \mathrm{ng} / \mathrm{ml}\left(1.6 \times 10^{-6} \mathrm{M}\right)$ is about 100 -fold greater than the concentration of RetAc employed in our studies, and t-RA was reported to be ineffective at lower concentrations.

Our c-neu antibody studies using the technique of IIF microscopy demonstrate that RetAc activates an erbB (c-neu) EGF-like receptor in post-mitotic suprabasal keratinocyte cells. In agreement, growth factor deprivation of NHK leads to a reduction in ERK phosphorylation, ${ }^{18-21}$ and is inhibited by neutralizing antibodies against amphiregulin and the ERK signaling pathway has also been implicated in hyperproliferative skin disorders and wound healing. ${ }^{22}$ Taken together, our results imply that RetAc activates $c$-neu-like receptors by translocation of cytoplasmic $c-n e u$ to membrane focal adhesion sites where the phosphorylation state is more stable, but can be further dephosphorylated by AP-treatment. Again, these results combined with the RTK1 loss of autocrine growth capacity in HaCaT-treated cells suggest that RetAc treatment activates $c$-neu-like receptors through altering the cellular location of the phosphorylation sites to target membrane associations.

Finally, our wound healing results show that physiological concentrations of RetAc do not impair the wound healing response in scratch wounded $\mathrm{HaCaT}$ monolayers, except at toxic concentrations. Healing under these conditions involves autocrine ligand production and activation of the erbB phosphorylation signaling pathway. These studies support our earlier disclosure, ${ }^{23}$ that keratinocyte cultures supplemented with insulin-like growth factor-1 (IGF-1) and RetAc in EGF-deficient SFM undergo stratified squamous keratinizing terminal differentiation suitable for preparation of an autologous cultured epidermal skin graft, ${ }^{24}$ and for other wound healing applications.

\section{Acknowledgements}

We wish to thank Dr. Mark Pittelkow of the Mayo Clinic for providing a sample RTK1(PD 98059). This work was completed at the Department of Biochemistry, Sloan Kettering Cancer Section of the University of Alabama at Birmingham, Al.

\section{Conflict of interest}

The author declares that there is no conflict of interest.

\section{References}

1. Amann PM, Schadendorf D, Owen RW, et al. Retinal and retinol are potential regulators of gene expression in the keratinocyte cell line HaCaT. Exp Dermatol. 2011;20(4):373-375.

2. DeLuca LM, Shapiro SS. Modulation of cellular interactions by vitamin A and derivatives (Retinoids). Ann NY Acad Sci.1981;359.

3. Boyce ST, Ham RG. Calcium regulated differentiation of normal human epidermal keratinocytes in chemically-defined clonal cultures and serumfree serial cultures. J Invest Dermatol. 1983;81(Suppl 1):33-40.

4. Wille JJ, Pittelkow MR, Shipley GD, et al. Integrated control of growth and differentiation of normal human prokeratinocytes cultured in serumfree medium: clonal analyses, growth kinetics and cell cycle studies. $J$ Cell Physiol. 1984;121(1):31-44.

5. Marcelo CL, Dunham WR. Retinoic acid stimulates essential fatty acid-supplemented human keratinocytes in culture. J Invest Dermatol. 1997;108(5):758-762.

6. Varani J, Nickoloff BJ, Dixit VM, et al. All-trans retinoic acid stimulates growth of adult human keratinocytes and fibroblasts cultured in growth factor-deficient medium, inhibits production of thrombospondin and fibronectin, and reduces adhesion. J Invest Dermatol. 1989;93(4):449-454.

7. Wille JJ, Park JY, Shealy YF. Cancer chemopreventive retinoids: validation and analysis of in vivo and in vitro bioassay results. $J$ Cancer Therapy. 2016;7(13):1008-1033.

8. Varani J, Nickoloff BJ, Dixit VM, et al. All-trans retinoic acid stimulates growth of adult human keratinocytes and fibroblasts cultured in growth factor-deficient medium, inhibits production of thrombospondin and fibronectin, and reduces adhesion. J Invest Dermatol. 1989;93(4):449-454.

9. Varani J, Burmeister B, Perone P, et al. All-trans retinoic acid inhibits fluctuations in intracellular $\mathrm{Ca}^{2+}$ resulting from changes in extracellylar $\mathrm{Ca}^{2+}$. Am J Pathol. 1995;147(3):718-727. 
10. Varani J, Zeigler M, Dame MK, et al. Heparin-binding epidermal-growthfactor-like growth factor activation of keratinocyteserbB receptors mediate epidermal hyperplasia, a prominent side-effect of retinoid therapy. J Invest Dermatol. 2001;117(6):1335-1341.

11. Wille JJ, Park JY. Effects of okadiac acid, retinoic acid, and phorbol myristate acetate tumor promoter on oncogene expression. J Cancer Ther. 2014;5(6):591-604

12. Wille JJ, Park JY. Retinoid and ethanol-sensitive benzo $(\alpha)$ pyrene induction of cytochrome P450 in human keratinocytes. $J$ Cancer Ther. 2012;3(6):1080-1085.

13. Wille JJ, Park JY. Induced differentiation of epitheliod carcinoma cell lines: evidence for tumor cell quantal mitosis. $J$ Cancer Therapy. 2016;7(11):794-811.

14. Wilke MS, Hsu BM, Wille JJ, et al. Biologic mechanisms for the regulation of normal human keratinocyte proliferation and differentiation. $A m J$ Pathol. 1988;131(1):171-181.

15. Wille JJ. Effect of antioxidants on the wound healing of keratinocyte monlayers. J Invest Dermatol. 2004;122(3):A71.

16. Cook PW, Mattox PA, Keeble WW, et al. A heparin sulfate-regulated human keratinocyte autocrine factor is similar or identical to amphiregulin. Mol Cell Biol. 1991;11(5):2547-2557.

17. Xiao JH, Feng X, Peng ZH, et al. Identification of heparin-binding EGFlike growth factor as a target in interecellular regulation of epidermal basal cell growth by suprabasal retinoic acid receptors. EMBO J. 1999;18(6):1539-1548.
18. Kansra S, Stoll SW, Johnson JL, et al. Autocrine ERK activation in normal keratinocytes: metalloproteinase-mediated release of amphiregulin triggers signaling from erbB1 to ERK. Mol Biol Cell. 2004;15(9):4299-309.

19. Kimura A, Terao M, Kato A, et al. Upregulation of $\mathrm{N}$-acetylglucosaminyltransferase- $\mathrm{V}$ by heparin-binding EGF-like growth factor induces keratinocyte proliferation and epidermal hyperplasia. Exp Dermatol. 2012;21(7):515-519.

20. Orechhia V, Regis G, Tassone B, et al. Constitutive STAT3 activation in epidermal keratinocytes enhances cell clonogenicity and favors spontaneous immortalization by opposing differentiation and senescence checkpoints. Exp Dermatol. 2015;24(1):29-34

21. Saeki Y, Nagashima T, Kimura S, et al. An erbB receptor-mediatede AP-1 regulatory network is modulated by STAT3 and c MYC during calcium-dependent keratinocyte differentiation. Exp Dermatol. 2012;21(4):293-298.

22. Rittie L, Varani J, Kang S, et al. Retinoid-induced epidermal hyperplasia is mediated by epidermal growth factor receptor activation via specific induction of its ligands heparin-binding EGF and amphiregulin in human skin in vivo. J Invest Dermatol. 2006;126(4):732-739.

23. Wille JJ. U.S. Patent No., 7,037,721. Protein-free defined media for the growth of normal human keratinocytes. 2006.

24. Wille JJ, Burdge JJ, Pittelkow MR. Rapid healing of chronic venous stasis leg ulcers treated by the application of a novel serum free cultured autologous epidermis. Wound Rep Reg. 2011;19(4):464-474. 